
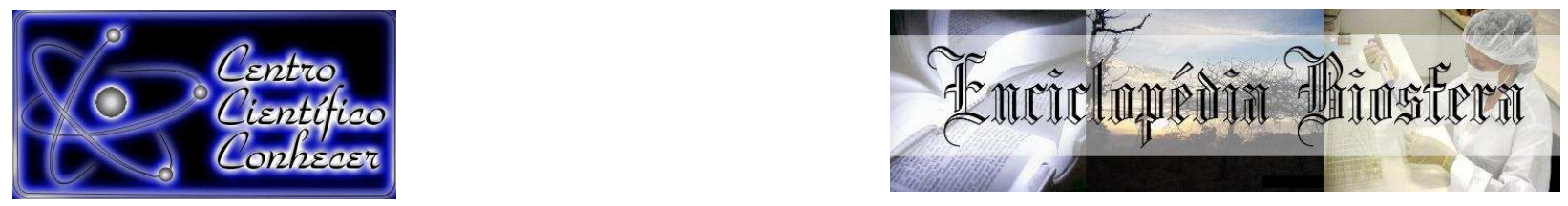

\title{
DISTRIBUIÇÃO ESPACIAL DE FORMIGAS CORTADEIRAS EM UM PLANTIO FLORESTAL DE Eucalyptus benthamii, NO MUNICÍPIO DE IRATI - PR
}

\footnotetext{
Luis Henrique da Silva ${ }^{1}$, Daniele Ukan²

1 Acadêmico de Engenharia Florestal da Universidade Estadual do Centro-Oeste, Irati, Paraná, Brasil (luish.engflorestal@hotmail.com).

2 Professora Doutora do curso de Engenharia Florestal da Universidade Estadual do Centro-Oeste, Irati, Paraná, Brasil.

Recebido em: 08/09/2015 - Aprovado em: 14/11/2015 - Publicado em: 01/12/2015

DOI: http://dx.doi.org/10.18677/Enciclopedia_Biosfera_2015_174
}

\begin{abstract}
RESUMO
Dentre as principais pragas florestais, ressaltam-se as formigas cortadeiras, que alcançaram o nível de pragas devido aos grandes prejuízos que causam aos povoamentos. Assim, o objetivo deste trabalho foi identificar as espécies de formigas cortadeiras em um plantio florestal de Eucalyptus benthamii no município de Irati/PR, localizado na região centro-oeste do Estado, também verificar a taxa de infestação de formigueiros na área, relatando o tamanho e a distribuição espacial dos formigueiros. A área experimental foi percorrida seguindo a linha de plantio, sendo contados e anotados todos os formigueiros visíveis, em cada formigueiro encontrado foram coletados exemplares de formigas para posterior identificação em laboratório. Foram estabelecidas três classes de tamanho para os ninhos de Acromyrmex: Classe I, com até $30 \mathrm{~cm}$ de diâmetro; classe II, entre 31 e $60 \mathrm{~cm}$; e classe III, com mais de $61 \mathrm{~cm}$. Para a determinação da distribuição espacial dos formigueiros dentro do talhão foi utilizado o índice de Morisita (I $\square$ ). Foram encontradas formigas cortadeiras apenas do gênero Acromyrmex, com predominância das espécies $A$. crassispinus e $A$. heyeri. O número de formigueiros identificados como de formigas cortadeiras encontrados na área foram de 0, 19 e 16, por classe, respectivamente. A distribuição dos formigueiros na área ocorre em agregados.
\end{abstract}

PALAVRAS-CHAVE: Acromyrmex, classes de diâmetro; pragas florestais

\section{IDENTIFICATION AND SPATIAL DISTRIBUTION OF LEAF-CUTTING ANTS IN A PLANTED FOREST OF Eucalyptus benthamii, IN IRATI - PR}

\begin{abstract}
Among the main forest plagues, we emphasize the leaf-cutting ants, which reached the level of plagues due to the extensive damage they cause to the stands. The aim of this work was to identify the species of leaf-cutting ants in a planted forest of Eucalyptus benthamii in the city of Irati/PR, located in the central-west region of the state, also to check the ant infestation rate in the area, reporting the size and the spatial distribution of anthills. The experimental area was covered following the planting row, being counted and recorded all visible anthills, in each nest found were collected specimens of ants for later identification in the laboratory. Three size class were established for the nests of Acromyrmex: Class I, with up to $30 \mathrm{~cm}$ in diameter; class II, between 31 and $60 \mathrm{~cm}$; and class III, with more than $61 \mathrm{~cm}$. To determine ENCICLOPÉDIA BIOSFERA, Centro Científico Conhecer - Goiânia, v.11 n.22; p.1456 2015
\end{abstract}


the spatial distribution of the nests within the stand it was used Morisita index (I $\square$ ). Leaf-cutting ants were found only the Acromyrmex genus, especially the species $A$. crassispinus and $A$. heyeri. The number of anthills identified as leaf-cutting ants found in the area were 0,19 and 16, by class, respectively. The distribution of the anthills in the area occurs in clusters.

KEYWORDS: Acromyrmex, forest plagues, diameter class.

\section{INTRODUÇÃO}

As formigas estão situadas dentro do Reino Animal, Filo Arthropoda, à Classe Insecta, Ordem Hymenoptera e Família Formicidae, Subfamília Myrmicinae, Tribo Attini. Quando se trata de formigas cortadeiras, este grupo de insetos é composto de 5 gêneros, sendo de maior importância econômica as do gênero Atta, conhecidas popularmente como saúva, e do gênero Acromyrmex, popularmente conhecidas como quenquém (JURUENA \& CACAHAPUZ, 1980).

A aparência externa dos ninhos de Atta e Acromyrmex pode ser utilizada para diferenciar os dois gêneros e auxiliar na identificação das espécies. Outro método utilizado envolve as diferenças morfológicas existentes entre esses dois gêneros (MOREIRA \& TONHASCA JUNIOR, 1998; LIMA et al., 2001). Segundo MICHELS et al., (2001), características físicas como dureza das folhas, presença de tricomas e presença de látex são fatores associados com a rejeição das formigas a determinadas plantas.

Segundo FERRONATO, (2013), devido aos danos frequentes que as formigas cortadeiras causam e a contínua presença de formigueiros em todas as fases de desenvolvimento florestal, estas precisam ser controladas. Para o controle desse inseto-praga os métodos mais utilizado pelas empresas são as iscas granuladas à base de fipronil e sulfluramida. Essas iscas, além de eficientes são de fácil aquisição no mercado, não apresentam dificuldades de aplicação e dispensam a localização dos formigueiros.

As vantagens apresentadas pela aplicação de iscas granuladas são evidentes, porém, existem dificuldades para se determinar a quantidade mais adequada para cada área antes da aplicação, proporcionando uma quantidade de iscas sem excessos, que seja eficaz para eliminar os formigueiros.

Para a determinação da quantidade correta de iscas a ser utilizada, baseia-se na identificação das espécies de ocorrência na área e no tamanho dos formigueiros. Assim, o este trabalho teve como objetivo principal identificar as espécies de formigas cortadeiras em um plantio florestal de Eucalyptus benthamii no município de Irati/PR, também verificar a taxa de infestação de formigueiros na área, relatando o tamanho e a distribuição espacial dos formigueiros.

\section{MATERIAL E MÉTODOS}

\section{Localização da área experimental}

O estudo foi realizado no Câmpus Universitário da Universidade Estadual do Centro-Oeste - UNICENTRO, município de Irati-PR, localizado a $156 \mathrm{~km}$ de Curitiba, entre as coordenadas 25 $27^{\prime}$ '56" sul e longitude $50^{\circ} 37^{\prime} 51^{\prime \prime}$ oeste. A avaliação foi realizada em uma área experimental com plantio de Eucalyptus benthamii, com aproximadamente 1 ha de área, quatro anos e meio de idade e espaçamento de $3 \mathrm{x}$ 2 metros. 
O clima da região, de acordo com a classificação de Köppen, recebe a denominação de subtropical $\mathrm{Cfb}$, o qual é caracterizado por meses de frio, com geadas severas e verões frescos, sem estação seca definida, com temperatura média no mês mais quente abaixo de $22^{\circ} \mathrm{C}$ e temperatura média no mês mais frio abaixo de $18^{\circ}$ (SEAB, 2010).

\section{Levantamento do grau de infestação dos formigueiros na área}

A área experimental foi percorrida seguindo a linha de plantio, sendo contados e anotados todos os formigueiros visíveis, nos quais foram obtidas as dimensões aparentes destes (maior comprimento x maior largura), como mostra a figura 1a. Foram coletados 5 exemplares de formigas de cada ninho para identificação e colocados em recipientes que foram etiquetados com o número do formigueiro e dia da coleta (Figura 1b). Em cada formigueiro foi marcado um ponto com o GPS, para facilitar a posterior localização dos mesmos e para que fosse possível a obtenção de um mapa dos formigueiros na área.
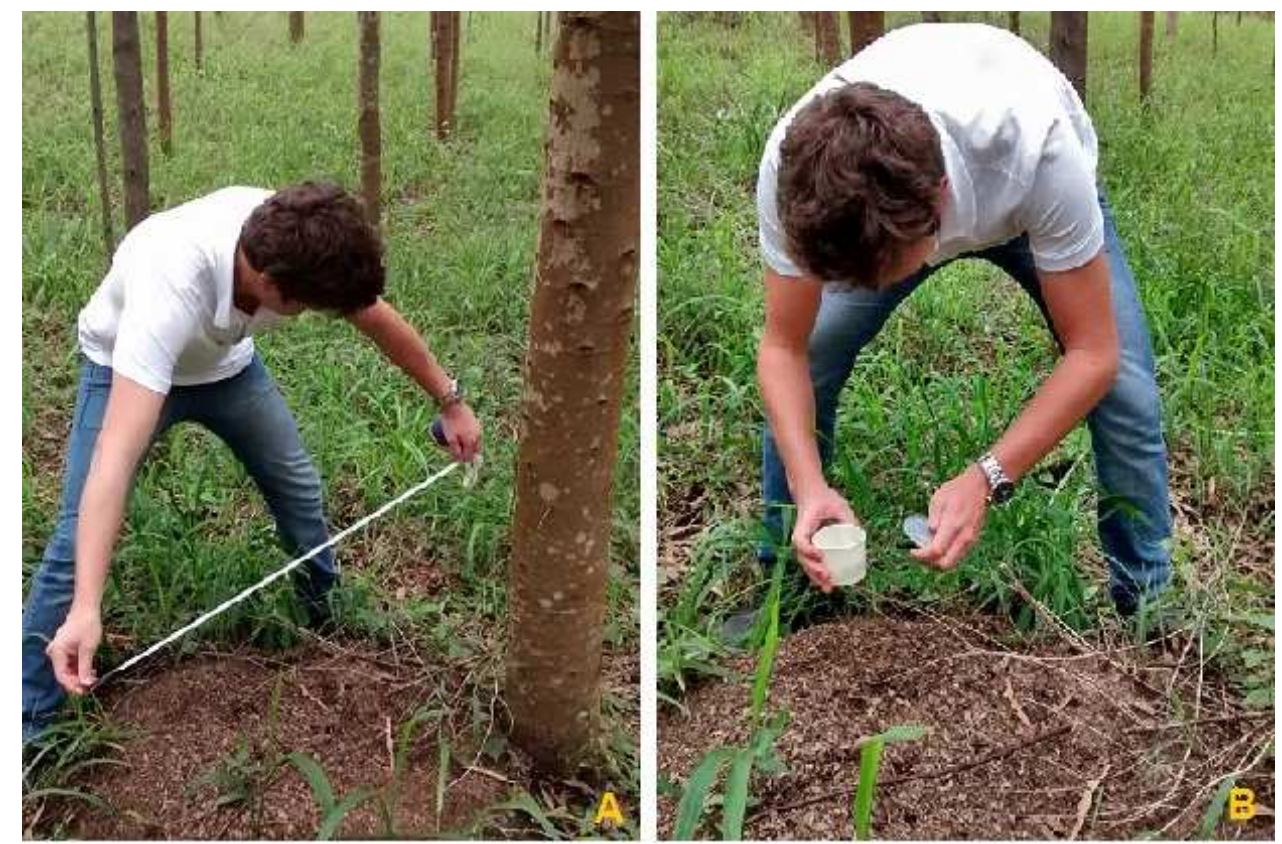

FIGURA 1. A- Medição da dimensão aparente dos formigueiros; BColeta de exemplares de formigas.

Após a coleta, todo o material foi levado para o laboratório de Proteção Florestal do Curso de Engenharia Florestal da Universidade Estadual do CentroOeste - UNICENTRO, Câmpus de Irati-PR, e foram acondicionados em vidros com álcool $70 \%$ para posteriormente serem montados e identificados. A identificação foi feita com o auxílio de uma lupa eletrônica, e foi realizada com base nas características anatômicas externas das formigas coletadas.

\section{Tamanho dos formigueiros e distribuição espacial}

Para a classificação do tamanho dos formigueiros o comprimento do maior eixo foi considerado como o diâmetro aproximado do ninho. Foram estabelecidas três classes de tamanho para os ninhos de Acromyrmex, seguindo a metodologia proposta por LINK et al., (2001): Classe I, com até $30 \mathrm{~cm}$ de diâmetro; classe II, entre 31 e $60 \mathrm{~cm}$; e classe III, com mais de $61 \mathrm{~cm}$. As classes foram estabelecidas 
para definir a frequência de formigueiros nas diferentes classes de tamanho. Segundo NICKELE et al., (2009), as diferentes classes de tamanho dos formigueiros apresentam relação com a idade, pois quanto mais velho o formigueiro, maior o seu tamanho.

Para a determinação da distribuição espacial dos formigueiros foi utilizado o índice de Morisita (I $\square$ ) (MORISITA, 1959). Este índice tem como critério de distribuição espacial: $I \square=1$, a distribuição dos formigueiros na área ocorre de forma aleatória; quando $I \square>1$, a distribuição é agregada; e $I \square<1$, a distribuição é regular (DAVIS, 1993).

$$
I_{\sigma}=N \frac{\sum x^{2}-\sum x}{\left(\sum x\right)^{2}-\sum x}
$$

Em que:

$\mathrm{N}=$ Número de unidades amostrais.

$\sum \mathrm{x}=$ Somatório dos indivíduos presentes nas unidades amostrais.

$\sum x^{2}=$ Somatório ao quadrado dos indivíduos presentes nas unidades amostrais.

A área total do terreno foi dividida em seis unidades amostrais, pois de acordo com BARROS \& MACHADO (1984), este índice é pouco influenciado pelo tamanho da unidade amostral e tem a vantagem de ser relativamente independente da média do número de amostras. Para o cálculo de distribuição espacial foram considerados apenas os formigueiros identificados como de formigas cortadeiras.

\section{RESULTADOS E DISCUSSÃO}

\section{Identificação das espécies encontradas na área}

Durante o levantamento foram encontrados 51 formigueiros em atividade na área de 1 ha. Destes, 35 foram identificados como do gênero Acromyrmex. Proporcionalmente, foram encontrados aproximadamente $69 \%$ de formigueiros de cortadeiras e $31 \%$ de não cortadeiras, confirmando a presença destes insetos nos reflorestamentos da região (Tabela 1 ).

TABELA 1. Formigueiros encontrados na área de estudos

\begin{tabular}{lcc}
\hline & \multicolumn{2}{c}{ Quantidade de formigueiros } \\
\cline { 2 - 3 } & $\mathrm{N}^{\circ}$ de formigueiros & $\%$ \\
\hline Cortadeiras & 35 & 68,62 \\
Não cortadeiras & 16 & 31,38 \\
\hline \multicolumn{1}{c}{ Total } & 51 & 100 \\
\hline
\end{tabular}

Dentro desse gênero foram encontrados duas espécies diferentes, $A$. crassispinus F. e $A$. heyeri F. Nos demais formigueiros, as formigas amostradas foram não foram identificados como formiga cortadeira e a identificação de gênero e/ou espécie não foi realizada.

Destas 35 colônias de formigas cortadeiras encontradas, houve predominância da espécie $A$. crassispinus, a qual foi identificada em 28 formigueiros, 
representando $80 \%$ do total. Já para a espécie $A$. heyeri foram identificados sete formigueiros, o que representou $20 \%$ do total de formigueiros de cortadeiras identificados (Tabela 2).

TABELA 2. Espécies identificadas como de formigas cortadeiras.

\begin{tabular}{ccc}
\hline \multirow{2}{*}{ Espécies } & \multicolumn{2}{c}{ Quantidade de formigueiros } \\
\cline { 2 - 3 } & $\mathrm{N}^{\circ}$ de formigueiros & $\%$ \\
\hline Acromyrmex crassispinus & 28 & 80 \\
Acromyrmex heyeri & 7 & 20 \\
\hline Total & 35 & 100 \\
\hline
\end{tabular}

Segundo BURATTO (2013) e DELLA LÚCIA et al., (1993), a espécie Acromyrmex crassispinus é a formiga cortadeira mais comum do sul do Brasil, também ocorre nos estados de São Paulo, Rio de Janeiro, Minas Gerais e Distrito Federal, é popularmente conhecida como "quenquém-de-cisco". A espécie Acromyrmex heyeri, conhecida popularmente como "formiga-de-monte-vermelha, tem distribuição nos estados: Rio Grande do Sul, Santa Catarina, Paraná e São Paulo (DELLA LÚCIA et al., 1993).

\section{Tamanho dos formigueiros e distribuição espacial}

$\mathrm{Na}$ área de estudo, houve predominância de formigueiros das classes II e III (Tabela 3), ou seja, formigueiros que já estavam instalados na área há algum tempo. Esse resultado coincide com o encontrado por NICKELE et al. (2009), relataram que em uma área com plantio com mais de três anos de idade ocorreu a predominância de formigueiros das classes II e III. Apenas dois formigueiros encontrados na área possuíram dimensão aparentemente maior que $1 \mathrm{~m}^{2}$.

TABELA 3. Número de formigueiros encontrados por classes de tamanho na área.

\begin{tabular}{cccc}
\hline & $\begin{array}{c}\text { Classe I } \\
\leq 30 \mathrm{~cm}\end{array}$ & $\begin{array}{c}\text { Classe II } \\
\text { de } 30 \text { a } 59 \mathrm{~cm}\end{array}$ & $\begin{array}{c}\text { Classe III } \\
\geq 60 \mathrm{~cm}\end{array}$ \\
\hline $\mathrm{N}^{\circ}$ de formigueiros & 0 & 19 & 16 \\
\hline
\end{tabular}

Segundo BURATTO (2013), as características do interior da floresta acabam sendo um dos principais problemas na dosagem correta de iscas a serem utilizadas para controle de formigas do gênero Acromyrmex, pois muitas vezes não se consegue determinar a densidade e a dimensão exata dos ninhos desse gênero.

As formigas do gênero Acromyrmex apresentam colônias menores que as do gênero Atta, por esse motivo a localização dos ninhos é mais difícil sobre a vegetação e restos da colheita florestal. Esse fato aumenta a importância sobre a necessidade de maiores estudos acerca do controle desta espécie (ZANETTI et al., 2003). A análise da distribuição espacial dos formigueiros, determinada pelo índice 
de dispersão de Morisita, demonstrou que a distribuição na área ocorre de forma agregada $(I \square=1,13)$ (Figura 2).

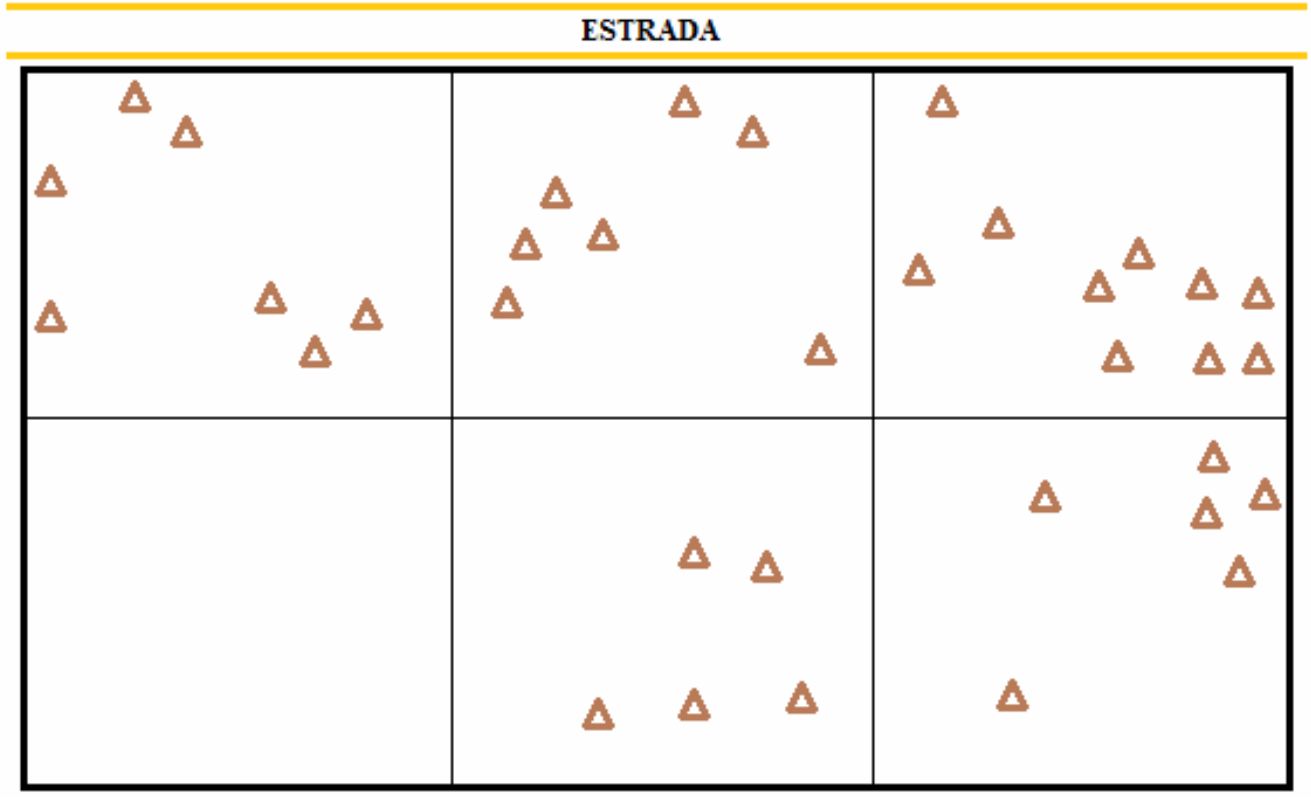

FIGURA 2. Distribuição espacial dos formigueiros na área de estudo.

Segundo ROGLIN et al., (2013), a distribuição dos formigueiros do tipo agregada pode ser resultado da distribuição de micro habitats, por interações mutualísticas ou por ramificações da colônia mãe, pois a heterogeneidade do ambiente influi de forma a ocorrer agregações de ninhos. Essa descrição coincide com o ambiente encontrado na área, que se trata de um plantio pequeno (1 ha) que não sofre tratos silviculturais, com isso ocorre competição entre as árvores levando a ocorrência de clareiras na área, onde observou-se uma grande agregação dos ninhos.

No entorno do plantio existem áreas de banhado, áreas de vegetação nativa e áreas de agricultura, nas proximidades dessas áreas foi possível notar a diferença no número de formigueiros, indicando a existência de interação entre as formigas e o ambiente.

\section{CONCLUSÕES}

Foram encontrados 51 formigueiros na área de estudo, sendo que 35 foram considerados colônias de formigas cortadeiras (68,62\%) e 16 foram consideradas como de formigas não-cortadeiras (31,38\%). Os levantamentos feitos na área indicaram formigas cortadeiras apenas do gênero Acromyrmex, com predominância das espécies $A$. crassispinus e $A$. heyeri.

A maioria dos formigueiros apresentaram área aparente entre 31 e $59 \mathrm{~cm}$ de diâmetro (Classe II), e apenas dois apresentaram área maior que $1 \mathrm{~m}^{2}$. A partir da análise da distribuição espacial dos formigueiros de formigas cortadeiras na área, verificou-se uma distribuição de forma agregada. Em função do número de formigueiros encontrados na área e aconselha-se a realização de controle destas formigas. 


\section{REFERÊNCIAS}

BARROS, P. L. C.; MACHADO, S. A. Aplicação de índices de dispersão em espécies de florestas tropicais da Amazônia brasileira. Série Científica I. Curitiba: FUPEF, 1984. 44p.

BURATTO, A. D. Uso de isca granuladas em plantações de Pinus taeda L., no planalto sul catarinense: Avaliação de consumo por formigas cortadeiras, formas de distribuição e degradação. 2013, 72 p. Dissertação (Mestrado em Engenharia Florestal) - Universidade Federal do Paraná, Curitiba, PR.

DAVIS, P. M. Statistics for describing populations, p.33-54. In: PEDIGO, L.P.; BUNTIN, G.D. Handbook of sampling methods for arthropods in agriculture. CRC Press, Boca Raton, 1993. 736p.

DELLA LUCIA, T. M. C.; E. F. VILELA. Métodos atuais de controle e perspectivas. p. 163 - 190. In: DELLA LUCIA, T. M. C. (Ed). As formigas cortadeiras. Vicosa: Folha de Vicosa, 1993.

FERRONATO, M. Z. Avaliação de dois sistemas de distribuição de iscas granuladas, à granel e em micro-porta-iscas, no controle de formigas cortadeiras. 2013, 72 p. Dissertação (Mestrado em Engenharia Florestal) Universidade Federal do Paraná, Curitiba, PR

JURUENA, L. F.; CACHAPUZ, L. M. M. Espécies de formigas cortadeiras ocorrentes no Estado do Rio Grande do Sul. Porto Alegre. Boletim de divulgação. Instituto de Pesquisas Agronômicas do Rio Grande do Sul, n²3, p 19-24, 1980.

LIMA, C. A., DELLA LUCIA, T. M. C., SILVA, N. A. Formigas cortadeiras: biologia e controle. Boletim de Extensão ํo 44. Viçosa, Minas Gerais, 2001. Universidade Federal de Viçosa.

LINK, D.; LINK, F. M.; OLIVEIRA, A. A. de. Potencial de dano da formiga preta, Acromyrmex crassispinus, em mudas de eucalipto e de pinus. In: SIMPÓSIO LATINO-AMERICANO SOBRE MANEJO FLORESTAL, 2001, Santa Maria. Anais... Santa Maria: Universidade Federal de Santa Maria, 2001. p. 671-676.

MICHELS, K.; CROMME, N.; GLATZLE, A.; SCHULTZE-KRAFT, R. Biological control of leaf-cutting ants using forage grasses: nest characteristics and fungus growth. Journal of Agronomy \& Crop Science, Hamilton, v. 187, p.259 - 267, 2001.

MOREIRA, D.; TONHASCA JUNIOR, A. Ecologia e controle de formigas cortadeiras. Campos dos Goytacazes, RJ: Universidade Estadual do Norte Fluminense. Boletim técnico, p 24, 1998.

MORISITA, M. Measuring of the dispersion of individuals and analysis of the distributional patterns. Memoirs of the Faculty of Science, Kyushi, v. 2, p. 215-235, 1959. 
NICKELE, M. A.; REIS FILHO, W; OLIVEIRA, B. E.; IEDE, T. E.; Densidade e tamanho de formigueiros de Acromyrmex crassispinus em plantios de Pinus taeda. Pesquisa Agropecuária Brasileira, Brasília, v. 44, n. 4, p. 347-353, 2009.

ROGLIN, A.; SOUSA, J. C.; PINTO, R. R. J.; KOEHLER S. H.; FERRONATO Z. N. Identificação e quantificação de formigas cortadeiras em áreas degradadas em processos de recuperação. Enciclopédia Biosfera, Goiânia, v. 9, n. 16, p. 1260, 2013.

SEAB. Secretaria de Agricultura e Abastecimento. Agrometeorologia (2010). Paraná, $2010 . \quad$ Disponível em: http://www.seab.pr.gov.br/arquivos/File/deral/Prognosticos/agrometeorologia 2009 10.pdf. Acesso em: 05/05/2015.

ZANETTI, R.; ZANUNCIO, J. C.; VILELA, E. F.; LEITE, H. G.; JAFFÉ, K.; OLIVEIRA, A. C. Level of economic damage for leaf-cutting ants (Hymenoptera: Formicidae) in Eucalyptus plantations in Brazil. Sociobiology, Chicio, v. 42, p. 433 - 444, 2003. 